\title{
Exploring uncertainties in dark energy constraints using current observational data with Planck 2015 distance priors
}

\author{
Yun Wang ${ }^{1,2, *}$ and Mi Dai ${ }^{2}$ \\ ${ }^{1}$ Infrared Processing and Analysis Center, California Institute of Technology, \\ 770 South Wilson Avenue, Pasadena, California 91125, USA \\ ${ }^{2}$ Homer L. Dodge Department of Physics \& Astronomy, University of Oklahoma, \\ 440 W Brooks Street, Norman, Oklahoma 73019, USA \\ (Received 9 September 2015; published 21 October 2016)
}

\begin{abstract}
We present the distance priors that we have derived from the 2015 Planck data, and use these in combination with the latest observational data from Type Ia supernovae (SNe Ia) and galaxy clustering, to explore the systematic uncertainties in dark energy constraints. We use the joint light-curve analysis (JLA) set of $740 \mathrm{SNe}$ Ia, galaxy clustering measurements of $H(z) s$ and $D_{A}(z) / s$ (where $s$ is the sound horizon at the drag epoch) from the Sloan Digital Sky Survey (SDSS) at $z=0.32$ and $z=0.57$ (BOSS DR12). We find that the combined dark energy constraints are insensitive to the assumptions made in the galaxy clustering measurements (whether they are for BAO only or marginalized over RSD), which indicates that as the analysis of galaxy clustering data becomes more accurate and robust, the systematic uncertainties are reduced. On the other hand, we find that flux-averaging SNe Ia at $z \geq 0.5$ significantly tightens the dark energy constraints and excludes a flat universe with a cosmological constant at $68 \%$ confidence level, assuming a dark energy equation of state linear in the cosmic scale factor. Flux averaging has the most significant effect when we allow the dark energy density function $X(z)$ to be a free function given by the cubic spline of its values at $z=0, \frac{1}{3}, \frac{2}{3}, 1$; the measured $X(z)$ deviates from a cosmological constant at more than $95 \%$ confidence level for $0.4 \lesssim z \lesssim 0.7$. Since flux averaging reduces the bias in the SN distance measurements, this may be an indication that we have arrived in the era when the SN distance measurements are limited by systematic uncertainties.
\end{abstract}

DOI: 10.1103/PhysRevD.94.083521

\section{INTRODUCTION}

We continue to search for the unknown cause for the observed cosmic acceleration [1,2], also known as dark energy. ${ }^{1}$ Current observational data offer tantalizing hints for deviations from a cosmological constant in a simplistic combination of all observational data without critical analysis. In order to arrive at robust constraints on dark energy, we must carefully examine all the data sets separately, and jointly. One complication is the difficulty of detecting and modeling unknown systematic uncertainties in the data used for the analysis.

In this paper, we explore the existence of unknown systematic uncertainties by critically analyzing the latest observational data from Type Ia supernovae (SNe Ia) and galaxy clustering, with the help of distance priors from cosmic microwave background anisotropy (CMB) data. We use the joint light-curve analysis (JLA) set of $740 \mathrm{SNe}$ Ia, galaxy clustering measurements of $H(z) s$ and $D_{A}(z) / s$ (where $s$ is the sound horizon at the drag epoch) from the Baryon Oscillation Spectroscopic Survey (BOSS) at $z=$ 0.32 and $z=0.57$, and the distance priors that we have derived from the 2015 Planck data.

\footnotetext{
*wang@ipac.caltech.edu

${ }^{1}$ For recent reviews, see Refs. [3-11].
}

We describe our method in Sec. II, present our results in Sec. III, and conclude in Sec. IV.

\section{METHOD}

We focus on exploring the unknown systematic uncertainties in the current SN Ia and galaxy clustering data using geometric constraints only, with distance priors from the 2015 Planck data to help tighten parameter constraints. For a conservative and transparent approach, we marginalize over constraints on the growth rate of cosmic largescale structure (which are degenerate with the geometric constraints $[12,13])$.

We now give the basic formulas that we will use later in the paper. The comoving distance to an object at redshift $z$ is given by

$$
\begin{gathered}
r(z)=c H_{0}^{-1}\left|\Omega_{k}\right|^{-1 / 2} \operatorname{sinn}\left[\left|\Omega_{k}\right|^{1 / 2} \Gamma(z)\right], \\
\Gamma(z)=\int_{0}^{z} \frac{d z^{\prime}}{E\left(z^{\prime}\right)}, \quad E(z)=H(z) / H_{0},
\end{gathered}
$$

where $\operatorname{sinn}(x)=\sin (x), x$, and $\sinh (x)$ for $\Omega_{k}<0, \Omega_{k}=0$, and $\Omega_{k}>0$, respectively. The Hubble parameter, $H(z)$, is given by 


$$
\begin{aligned}
H^{2}(z) \equiv & \left(\frac{\dot{a}}{a}\right)^{2} \\
= & H_{0}^{2}\left[\Omega_{m}(1+z)^{3}+\Omega_{r}(1+z)^{4}\right. \\
& \left.+\Omega_{k}(1+z)^{2}+\Omega_{X} X(z)\right],
\end{aligned}
$$

where $\Omega_{m}+\Omega_{r}+\Omega_{k}+\Omega_{X}=1$. The dark energy density function $X(z) \equiv \rho_{X}(z) / \rho_{X}(0)$. The $\Omega_{r}$ term, with $\Omega_{r}=$ $\Omega_{m} /\left(1+z_{\text {eq }}\right) \ll \Omega_{m}$ ( $z_{\text {eq }}$ denotes the redshift at matterradiation equality), is usually omitted in dark energy studies at $z \ll 1000$, since dark energy should only be important at late times. For comparison with the work of others and to provide a reference for future surveys, we consider a dark energy equation of state linear in the cosmic scale factor $a$ [14]:

$$
w_{X}(a)=w_{0}+(1-a) w_{a} .
$$

In addition, we consider an alternative two-parameter parametrization of $w_{X}(a)$, using $w_{0}$ and $w_{0.5} \equiv w_{X}(z=0.5)$ :

$$
\begin{aligned}
w_{X}(a) & =\left(\frac{\frac{2}{3}-a}{\frac{2}{3}-1}\right) w_{0}+\left(\frac{a-1}{\frac{2}{3}-1}\right) w_{0.5} \\
& =3 w_{0.5}-2 w_{0}+3\left(w_{0}-w_{0.5}\right) a .
\end{aligned}
$$

Note that $a=\frac{2}{3}$ for $z=0.5$. It has been shown that $\left(w_{0}, w_{0.5}\right)$ are significantly less correlated compared to $\left(w_{0}, w_{a}\right)[15]$.

Finally, we consider a model-independent parametrization of $X(z)$, where $X(z)$ is a free function of redshift given by the cubic spline of its value at $z=0, \frac{1}{3}, \frac{2}{3}$, and 1 . We assume that $X(z>1)=X(z=1)$.

\section{A. CMB data}

We use CMB data in the condensed form of the CMB shift parameters (also known as distance priors) [16]:

$$
\begin{aligned}
& R \equiv \sqrt{\Omega_{m} H_{0}^{2}} r\left(z_{*}\right) / c, \\
& l_{a} \equiv \pi r\left(z_{*}\right) / r_{s}\left(z_{*}\right) .
\end{aligned}
$$

These two parameters, $R$ and $l_{a}$, together with $\omega_{b} \equiv \Omega_{b} h^{2}$, provide an efficient summary of CMB data as far as dark energy constraints go $[16,17]$.

CMB data give us the comoving distance to the photondecoupling surface $r\left(z_{*}\right)$ and the comoving sound horizon at the photon-decoupling epoch $r_{s}\left(z_{*}\right)$ [18]. The comoving sound horizon at redshift $z$ is given by

$$
\begin{aligned}
r_{s}(z) & =\int_{0}^{t} \frac{c_{s} d t^{\prime}}{a}=c H_{0}^{-1} \int_{z}^{\infty} d z^{\prime} \frac{c_{s}}{E\left(z^{\prime}\right)}, \\
& =c H_{0}^{-1} \int_{0}^{a} \frac{d a^{\prime}}{\sqrt{3\left(1+\overline{R_{b}} a^{\prime}\right) a^{\prime 4} E^{2}\left(z^{\prime}\right)}},
\end{aligned}
$$

where $a$ is the cosmic scale factor, $a=1 /(1+z)$, and $a^{4} E^{2}(z)=\Omega_{m}\left(a+a_{\mathrm{eq}}\right)+\Omega_{k} a^{2}+\Omega_{X} X(z) a^{4}$, with $a_{\mathrm{eq}}=\Omega_{\mathrm{rad}} / \Omega_{m}=1 /\left(1+z_{\mathrm{eq}}\right)$ and $z_{\mathrm{eq}}=2.5 \times 10^{4} \Omega_{m} \times$ $h^{2}\left(T_{\mathrm{CMB}} / 2.7 \mathrm{~K}\right)^{-4}$. The sound speed is $c_{s}=$ $1 / \sqrt{3\left(1+\overline{R_{b}} a\right)}$, with $\overline{R_{b}} a=3 \rho_{b} /\left(4 \rho_{\gamma}\right)$ and $\overline{R_{b}}=$ $31500 \Omega_{b} h^{2}\left(T_{\mathrm{CMB}} / 2.7 \mathrm{~K}\right)^{-4}$. We take $T_{\mathrm{CMB}}=2.7255$.

The redshift to the photon-decoupling surface, $z_{*}$, is given by the fitting formula [19]:

$z_{*}=1048\left[1+0.00124\left(\Omega_{b} h^{2}\right)^{-0.738}\right]\left[1+g_{1}\left(\Omega_{m} h^{2}\right)^{g_{2}}\right]$,

where

$$
\begin{aligned}
& g_{1}=\frac{0.0783\left(\Omega_{b} h^{2}\right)^{-0.238}}{1+39.5\left(\Omega_{b} h^{2}\right)^{0.763}}, \\
& g_{2}=\frac{0.560}{1+21.1\left(\Omega_{b} h^{2}\right)^{1.81}} .
\end{aligned}
$$

The redshift of the drag epoch $z_{d}$ is well approximated by [20]

$$
z_{d}=\frac{1291\left(\Omega_{m} h^{2}\right)^{0.251}}{1+0.659\left(\Omega_{m} h^{2}\right)^{0.828}}\left[1+b_{1}\left(\Omega_{b} h^{2}\right)^{b 2}\right]
$$

where

$$
\begin{aligned}
& b_{1}=0.313\left(\Omega_{m} h^{2}\right)^{-0.419}\left[1+0.607\left(\Omega_{m} h^{2}\right)^{0.674}\right], \\
& b_{2}=0.238\left(\Omega_{m} h^{2}\right)^{0.223} .
\end{aligned}
$$

Since the constraints on $\left(l_{a}, R, \omega_{b}, n_{s}\right)$ are not sensitive to the assumption about dark energy [21], we are able to use the Planck archive to obtain constraints on $\left(l_{a}, R, \omega_{b}, n_{s}\right)$ from the 2015 Planck data. We use data from the Planck archive that include both temperature and polarization data, as well as CMB lensing. As we have shown in earlier work [16], the one-dimensional marginalized probability distributions of $\left(l_{a}, R, \omega_{b}, n_{s}\right)$ are well fitted by Gaussian distributions. For the Planck 2015 data, $\left(l_{a}, R, \omega_{b}, n_{s}\right)$ are given by Gaussian distributions with the following means and standard deviations, without assuming a flat universe:

$$
\begin{array}{rlrl}
\left\langle l_{a}\right\rangle & =301.76, \quad \sigma\left(l_{a}\right)=0.093, \\
\langle R\rangle & =1.7474, & \sigma(R)=0.0051, \\
\left\langle\omega_{b}\right\rangle & =0.02228, & \sigma\left(\omega_{b}\right)=0.00016, \\
\left\langle n_{s}\right\rangle & =0.9659, \quad \sigma\left(n_{s}\right)=0.0048,
\end{array}
$$

with the normalized covariance matrix of $\left(l_{a}, R, \omega_{b}, n_{s}\right)$ : 


$$
\left(\begin{array}{cccc}
1.0000 & 0.4529 & -0.3507 & -0.3576 \\
0.4529 & 1.0000 & -0.7000 & -0.7780 \\
-0.3507 & -0.7000 & 1.0000 & 0.5296 \\
-0.3576 & -0.7780 & 0.5296 & 1.0000
\end{array}\right) .
$$

Assuming a flat universe, the Planck 2015 data give $\left(l_{a}, R, \omega_{b}, n_{s}\right)$ well fit by Gaussian distributions with the following means and standard deviations:

$$
\begin{array}{rlrl}
\left\langle l_{a}\right\rangle & =301.77, & \sigma\left(l_{a}\right)=0.090, \\
\langle R\rangle & =1.7482, & \sigma(R)=0.0048, \\
\left\langle\omega_{b}\right\rangle & =0.02226, & \sigma\left(\omega_{b}\right)=0.00016, \\
\left\langle n_{s}\right\rangle & =0.9653, & \sigma\left(n_{s}\right) & =0.0048,
\end{array}
$$

with the normalized covariance matrix of $\left(l_{a}, R, \omega_{b}, n_{s}\right)$ :

$$
\left(\begin{array}{cccc}
1.0000 & 0.3996 & -0.3181 & -0.3004 \\
0.3996 & 1.0000 & -0.6891 & -0.7677 \\
-0.3181 & -0.6891 & 1.0000 & 0.5152 \\
-0.3004 & -0.7677 & 0.5152 & 1.0000
\end{array}\right)
$$

We have included $n_{s}$ in our distance priors for completeness. For the remainder of this paper, we marginalize the CMB distance priors over $n_{s}$. This means dropping the fourth row and fourth column from the normalized covariance matrix of $\left(l_{a}, R, \omega_{b}, n_{s}\right)$, then obtaining the covariance matrix for $\left(l_{a}, R, \omega_{b}\right)$ as follows:

$$
\operatorname{Cov}_{\mathrm{CMB}}\left(p_{i}, p_{j}\right)=\sigma\left(p_{i}\right) \sigma\left(p_{j}\right) \operatorname{NormCov}_{\mathrm{CMB}}\left(p_{i}, p_{j}\right),
$$

where $i, j=1,2,3$. The rms variance $\sigma\left(p_{i}\right)$ and the normalized covariance matrix $\operatorname{NormCov}_{\mathrm{CMB}}$ are given by Eqs. (13) and (14) without assuming a flat universe, and by Eqs. (15) and (16) for a flat universe.

We include the Planck distance priors by adding the following term to the $\chi^{2}$ of a given model with $p_{1}=l_{a}$, $p_{2}=R$, and $p_{3}=\omega_{b}$ :

$$
\begin{aligned}
\chi_{\mathrm{CMB}}^{2} & =\Delta p_{i}\left[\operatorname{Cov}_{\mathrm{CMB}}^{-1}\left(p_{i}, p_{j}\right)\right] \Delta p_{j}, \\
\Delta p_{i} & =p_{i}-p_{i}^{\text {data }}
\end{aligned}
$$

where $p_{i}^{\text {data }}$ are the means from Eq. (13) (without assuming a flat universe) and Eq. (15) (assuming a flat universe), and $\operatorname{Cov}_{\mathrm{CMB}}^{-1}$ is the inverse of the covariance matrix of $\left[l_{a}, R, \omega_{b}\right]$ from Eq. (17). Note that $p_{4}=n_{s}$ should be added if the constraints on $n_{s}$ are included in the galaxy clustering data.

\section{B. Analysis of SN Ia data}

The distance modulus to a SN Ia is given by

$$
\mu_{0} \equiv m-M=5 \log \left[\frac{d_{L}(z)}{\mathrm{Mpc}}\right]+25,
$$

where $m$ and $M$ represent the apparent and absolute magnitudes of a SN. The luminosity distance is $d_{L}(z)=$ $(1+z) r(z)$, with the comoving distance $r(z)$ given by Eq. (1).

We use the JLA set of 740 SNe Ia processed by Betoule et al. (2014) [22]. They give the apparent $B$ magnitude, $m_{B}$, and the covariance matrix for $\Delta m \equiv m_{B}-m_{\text {mod }}$, with [23]

$$
m_{\text {mod }}=5 \log _{10} \mathcal{D}_{L}(z \mid \mathbf{s})-\alpha X_{1}+\beta \mathcal{C}+\mathcal{M},
$$

where $\mathcal{D}_{L}(z \mid \mathbf{s})$ is the luminosity distance multiplied by $H_{0}$ for a given set of cosmological parameters $\{\mathbf{s}\}, X_{1}$ is the stretch measure of the $\mathrm{SN}$ light-curve shape, and $\mathcal{C}$ is the color measure for the $\mathrm{SN}$. $\mathcal{M}$ is a nuisance parameter representing some combination of the absolute magnitude of a fiducial SN Ia, $M$, and the Hubble constant $H_{0} . \mathcal{M}$ is assumed to be different for SNe Ia with different host stellar masses:

$$
\begin{aligned}
& \mathcal{M}=M_{1} \text { for host stellar mass }<10^{10} M_{\odot} \\
& \mathcal{M}=M_{2} \text { otherwise. }
\end{aligned}
$$

Since the time dilation part of the observed luminosity distance depends on the total redshift $z_{\text {hel }}$ (special relativistic plus cosmological), we have [24]

$$
\mathcal{D}_{L}(z \mid \mathbf{s}) \equiv c^{-1} H_{0}\left(1+z_{\text {hel }}\right) r(z \mid \mathbf{s}),
$$

where $z$ and $z_{\text {hel }}$ are the CMB rest frame and heliocentric redshifts of the $\mathrm{SN}$.

For a set of $N \mathrm{SNe}$ with correlated errors, we have

$$
\chi^{2}=\Delta \mathbf{m}^{T} \cdot \mathbf{C}^{-1} \cdot \Delta \mathbf{m},
$$

where $\Delta \mathbf{m}$ is a vector with $N$ components, and $\mathbf{C}$ is the $N \times N$ covariance matrix of the $\mathrm{SNe}$ Ia.

Note that $\Delta m$ is equivalent to $\Delta \mu_{0}$, since

$$
\Delta m \equiv m_{B}-m_{\text {mod }}=\left[m_{B}+\alpha X_{1}-\beta \mathcal{C}\right]-\mathcal{M} .
$$

The total covariance matrix is [23]

$$
\mathbf{C}=\mathbf{D}_{\text {stat }}+\mathbf{C}_{\text {stat }}+\mathbf{C}_{\text {sys }},
$$

with the diagonal part of the statistical uncertainty given by $[22,23]$ 


$$
\begin{aligned}
\mathbf{D}_{\text {stat }, \mathrm{ii}}= & \sigma_{m_{B}, i}^{2}+\sigma_{\text {int }}^{2}+\sigma_{\text {lensing }}^{2}+\left[\frac{5}{z_{i} \ln 10}\right]^{2} \sigma_{z, i}^{2} \\
& +\alpha^{2} \sigma_{X_{1}, i}^{2}+\beta^{2} \sigma_{\mathcal{C}, i}^{2}+2 \alpha C_{m_{B} X_{1}, i}-2 \beta C_{m_{B} \mathcal{C}, i} \\
& -2 \alpha \beta C_{X_{1} \mathcal{C}, i}
\end{aligned}
$$

where $C_{m_{B} X_{1}, i}, C_{m_{B} \mathcal{C}, i}$, and $C_{X_{1} \mathcal{C}, i}$ are the covariances between $m_{B}, X_{1}$, and $\mathcal{C}$ for the $i$ th $\mathrm{SN}$. Note that Betoule et al. (2014) included host galaxy correction in $\mathbf{C}_{\text {stat }}+\mathbf{C}_{\text {sys }}$ [see Eq. (11) of Ref. [22]].

The statistical and systematic covariance matrices, $\mathbf{C}_{\text {stat }}$ and $\mathbf{C}_{\text {sys }}$, are generally not diagonal [23], and are given in the form

$$
\begin{aligned}
\mathbf{C}_{\text {stat }}+\mathbf{C}_{\text {sys }}= & V_{0}+\alpha^{2} V_{a}+\beta^{2} V_{b}+2 \alpha V_{0 a} \\
& -2 \beta V_{0 b}-2 \alpha \beta V_{a b},
\end{aligned}
$$

where $V_{0}, V_{a}, V_{b}, V_{0 a}, V_{0 b}$, and $V_{a b}$ are matrices given by Betoule et al. at the link http://supernovae.in2p3.fr/ sdss_snls_jla/ReadMe.html, and $\mathbf{C}_{\text {stat }}$ includes the uncertainty in the SN model. $\mathbf{C}_{\text {sys }}$ includes the uncertainty in the zero point. Note that $\mathbf{C}_{\text {stat }}$ and $\mathbf{C}_{\text {sys }}$ do not depend on $\mathcal{M}$, since the relative distance moduli are independent of the value of $\mathcal{M}$ [23].

We refer the reader to Conley et al. (2011) [23] and Betoule et al. (2014) [22] for detailed discussions of the origins of the statistical and systematic errors.

In order to explore the existence of unknown systematic effects, we apply flux averaging to the JLA SNe Ia at $z \geq 0.5$. Flux averaging was proposed to reduce the systematic bias in distance measurement due to weak lensing magnification of SNe Ia [25-27]; it has the additional benefit of reducing the bias in distance estimates due to other, possibly unknown, systematic effects [28]. This is because flux averaging effectively reduces a global systematic bias to a local bias with a much smaller amplitude, which in turn results in a reduced impact on global parameter constraints. Since weak lensing does not have a significant effect on SN Ia data (see, e.g., Ref. [29]), any systematic biases in the current SN Ia data are likely dominated by other, presently unknown, sources.

Here we apply flux averaging in the minimal approach of flux-averaging the SNe Ia in each redshift bin at higher $z$, and then use the usual "magnitude statistics" (instead of "flux statistics" [25-27]) in computing $\chi^{2}$, since the JLA $\mathrm{SNe}$ Ia have measurement and modeling errors that have been effectively Gaussianized in magnitudes.

For $\chi^{2}$ statistics using Markov Chain Monte Carlo (MCMC) or a grid of parameters, here are the steps in flux averaging [21] in application to the JLA SNe Ia:

1. Convert the distance modulus of $\mathrm{SNe} \mathrm{Ia}$ into "fluxes":

$$
F\left(z_{l}\right) \equiv 10^{-\left(\mu_{0}^{\text {data }}\left(z_{l}\right)-25\right) / 2.5}=\left(\frac{d_{L}^{\text {data }}\left(z_{l}\right)}{\mathrm{Mpc}}\right)^{-2} .
$$

2. For a given set of cosmological parameters $\{\mathbf{s}\}$, obtain "absolute luminosities" $\left\{\mathcal{L}\left(z_{l}\right)\right\}$ by removing the redshift dependence of the "fluxes"; i.e.,

$$
\mathcal{L}\left(z_{l}\right) \equiv d_{L}^{2}\left(z_{l} \mid \mathbf{s}\right) F\left(z_{l}\right) .
$$

3. Flux-average the "absolute luminosities" $\left\{\mathcal{L}_{l}^{i}\right\}$ in each redshift bin $i$ to obtain $\left\{\overline{\mathcal{L}}^{i}\right\}$ :

$$
\overline{\mathcal{L}}^{i}=\frac{1}{N_{i}} \sum_{l=1}^{N_{i}} \mathcal{L}_{l}^{i}\left(z_{l}^{(i)}\right), \quad \overline{z_{i}}=\frac{1}{N_{i}} \sum_{l=1}^{N_{i}} z_{l}^{(i)} .
$$

4. Place $\overline{\mathcal{L}}^{i}$ at the mean redshift $\bar{z}_{i}$ of the $i$ th redshift bin; now the binned flux is

$$
\bar{F}\left(\bar{z}_{i}\right)=\overline{\mathcal{L}}^{i} / d_{L}^{2}\left(\bar{z}_{i} \mid \mathbf{s}\right),
$$

with the corresponding flux-averaged distance modulus

$$
\bar{\mu}^{\mathrm{data}}\left(\bar{z}_{i}\right)=-2.5 \log _{10} \bar{F}\left(\bar{z}_{i}\right)+25 .
$$

5. Compute the covariance matrix of $\bar{\mu}\left(\bar{z}_{i}\right)$ and $\bar{\mu}\left(\bar{z}_{j}\right)$ :

$$
\begin{aligned}
\operatorname{Cov}\left[\bar{\mu}\left(\bar{z}_{i}\right), \bar{\mu}\left(\bar{z}_{j}\right)\right] \\
=\frac{1}{N_{i} N_{j} \overline{\mathcal{L}^{i} \overline{\mathcal{L}}^{j}}} \\
\quad \cdot \sum_{l=1}^{N_{i}} \sum_{m=1}^{N_{j}} \mathcal{L}\left(z_{l}^{(i)}\right) \mathcal{L}\left(z_{m}^{(j)}\right)\left\langle\Delta \mu_{0}^{\text {data }}\left(z_{l}^{(i)}\right) \Delta \mu_{0}^{\text {data }}\left(z_{m}^{(j)}\right)\right\rangle,
\end{aligned}
$$

where $\left\langle\Delta \mu_{0}^{\text {data }}\left(z_{l}^{(i)}\right) \Delta \mu_{0}^{\text {data }}\left(z_{m}^{(j)}\right)\right\rangle$ is the covariance of the measured distance moduli of the $l$ th SN Ia in the $i$ th redshift bin, and the $m$ th SN Ia in the $j$ th redshift bin. $\mathcal{L}(z)$ is defined by Eqs. (28) and (29).

6. For the flux-averaged data $\left\{\bar{\mu}\left(\bar{z}_{i}\right)\right\}$, compute

$$
\chi^{2}=\sum_{i j} \Delta \bar{\mu}\left(\bar{z}_{i}\right) \operatorname{Cov}^{-1}\left[\bar{\mu}\left(\bar{z}_{i}\right), \bar{\mu}\left(\bar{z}_{j}\right)\right] \Delta \bar{\mu}\left(\bar{z}_{j}\right)
$$

where

$$
\Delta \bar{\mu}\left(\bar{z}_{i}\right) \equiv \bar{\mu}\left(\bar{z}_{i}\right)-\mu^{p}\left(\bar{z}_{i} \mid \mathbf{s}\right)
$$

and

$$
\bar{\mu}^{p}\left(\bar{z}_{i}\right)=-2.5 \log _{10} F^{p}\left(\bar{z}_{i}\right)+25,
$$

with $F^{p}\left(\bar{z}_{i} \mid \mathbf{s}\right)=\left(d_{L}(z \mid \mathbf{s}) / \mathrm{Mpc}\right)^{-2}$. 
For the sample of SNe we use in this study, we fluxaveraged the $\mathrm{SNe}$ with $d z=0.04$.

\section{Galaxy clustering data}

For galaxy clustering data, we use the measurements of $x_{h}(z)=H(z) r_{s}\left(z_{d}\right) / c$ and $x_{d}(z)=D_{A}(z) / r_{s}\left(z_{d}\right)$, where $H(z)$ is the Hubble parameter, $D_{A}(z)$ is the angular diameter distance, and $r_{s}\left(z_{d}\right)$ is the sound horizon at the drag epoch. It has been shown that $x_{h}(z)$ and $x_{d}(z)$ are more tightly constrained by data and less sensitive to modeling assumptions, compared to $H(z)$ and $D_{A}(z)$ [30]. We use the $x_{h}(z)$ and $x_{d}(z)$ measurements from the two-dimensional power spectrum measured at $z=0.32$ and $z=0.57$ from BOSS DR12 galaxies [31,32]. Converting the results in Refs. [31,32] to the same definitions used in this paper, we find that for BAO only [31],

$$
\begin{aligned}
x_{h}(0.32) \equiv H(0.32) r_{s}\left(z_{d}\right) / c & =0.0397 \pm 0.0021 \\
x_{d}(0.32) \equiv D_{A}(0.32) / r_{s}\left(z_{d}\right) & =6.49 \pm 0.16 \\
r_{h d}(0.32) & =0.41
\end{aligned}
$$

$x_{h}(0.57) \equiv H(0.57) r_{s}\left(z_{d}\right) / c=0.0498 \pm 0.0013$,

$x_{d}(0.57) \equiv D_{A}(0.57) / r_{s}\left(z_{d}\right)=9.18 \pm 0.13$,

$$
r_{h d}(0.57)=0.47 \text {. }
$$

For BAO measurements marginalized over RSD [32], we find

$$
\begin{aligned}
x_{h}(0.32) \equiv H(0.32) r_{s}\left(z_{d}\right) / c & =0.0391 \pm 0.0019, \\
x_{d}(0.32) \equiv D_{A}(0.32) / r_{s}\left(z_{d}\right) & =6.185 \pm 0.185, \\
r_{h d}(0.32) & =0.5 ;
\end{aligned}
$$

$x_{h}(0.57) \equiv H(0.57) r_{s}\left(z_{d}\right) / c=0.0476 \pm 0.0015$,

$x_{d}(0.57) \equiv D_{A}(0.57) / r_{s}\left(z_{d}\right)=9.18 \pm 0.15$,

$$
r_{h d}(0.57)=0.53 \text {. }
$$

Galaxy clustering data are included in our analysis by adding $\chi_{G C}^{2}=\chi_{G C 1}^{2}+\chi_{G C 2}^{2}$, with $z_{G C 1}=0.32$ and $z_{G C 2}=0.57$, to the $\chi^{2}$ of a given model. Note that

$\chi_{G C i}^{2}=\Delta p_{i}\left[\mathbf{C}_{G C}^{-1}\left(p_{i}, p_{j}\right)\right] \Delta p_{j}, \quad \Delta p_{i}=p_{i}-p_{i}^{\text {data }}$,

where $p_{1}=H\left(z_{G C i}\right) r_{s}\left(z_{d}\right) / c$ and $p_{2}=D_{A}\left(z_{G C i}\right) / r_{s}\left(z_{d}\right)$, with $i=1,2$.

\section{RESULTS}

We perform an MCMC likelihood analysis [33] to obtain $\mathcal{O}\left(10^{6}\right)$ samples for each set of results presented in this paper. We assume flat priors for all the parameters, and we allow ranges of the parameters wide enough such that further increasing the allowed ranges has no impact on the results. We constrain dark energy and cosmological parameters $\left(w_{0}, w_{a}, \Omega_{m}, \Omega_{k}, h, \omega_{b}\right)$, where $\omega_{b} \equiv \Omega_{b} h^{2}$. In addition, we marginalize over the SN Ia nuisance parameters $\left\{\alpha, \beta, M_{1}, M_{2}\right\}$.

\section{A. Constraints on $w_{0}$ and $w_{a}$}

Figure 1 shows the marginalized probability distributions of parameters from JLA SNe, galaxy clustering data at
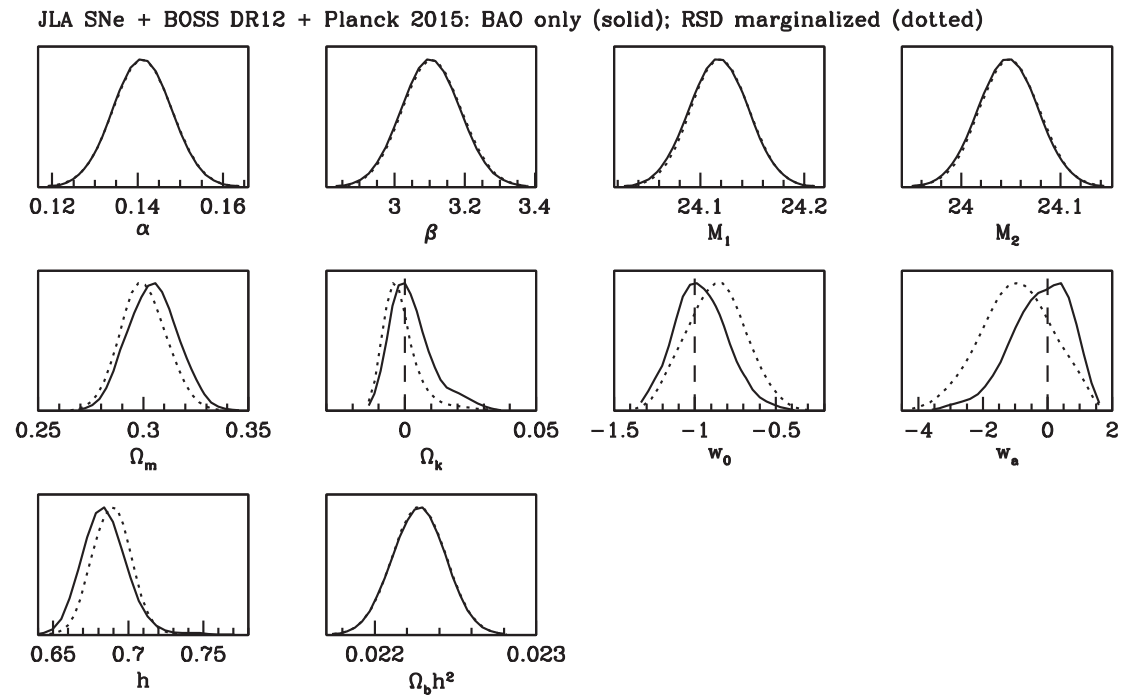

FIG. 1. Marginalized probability distributions of parameters from JLA SNe, galaxy clustering data at $z=0.32$ and $z=0.57$, and Planck 2015 distance priors. The solid and dotted curves correspond to using $H(z)$ and $D_{A}(z)$ measurements from BAO-only measurements, and using those from RSD marginalized measurements. 
$z=0.32$ and $z=0.57$ [31,32], and Planck 2015 distance priors presented in this paper (see Sec. II A). The solid and dotted curves correspond to using $H(z)$ and $D_{A}(z)$ measurements from BAO-only measurements, and using those from RSD marginalized measurements. Figure 2 shows the joint $68 \%$ and $95 \%$ confidence contours for $\left(w_{a}, w_{0}\right)$ and $\left(w_{a}, \Omega_{k}\right)$ corresponding to Fig. 1, with the same line types. The combined dark energy constraints seem insensitive to the assumptions made in the analysis of galaxy clustering data.

Figure 3 shows the impact of flux-averaging SNe Ia on the marginalized probability distributions of parameters from the combination of the same data sets as in Fig. 1. The solid and dotted curves correspond to using SNe Ia with and without flux averaging. Figure 4 shows the joint $68 \%$ and $95 \%$ confidence contours for $\left(w_{a}, w_{0}\right)$ and $\left(w_{a}, \Omega_{k}\right)$ corresponding to Fig. 3, with the same line types. Clearly, flux averaging significantly tightens the dark energy constraints. This may be due to the reduction in the bias of distance measurements from flux averaging, which increases the concordance of the data, resulting in tighter constraints.

\section{B. Constraints on $w_{0}$ and $w_{0.5}$}

Figures 5-8 are similar to Figs. 1-4, but for parametrizing the linear dark energy density using $w_{0}$ and $w_{0.5}$ [see Eq. (4)], instead of the usual $w_{0}$ and $w_{a}$. Figure 5 shows the impact of the galaxy clustering analysis technique on the marginalized probability distributions of parameters from JLA SNe, galaxy clustering data at $z=$ 0.32 and $z=0.57$ [31,32], and Planck 2015 distance priors presented in this paper (see Sec. II A). The solid and dotted curves correspond to using $H(z)$ and $D_{A}(z)$ measurements from BAO-only measurements, and using those from RSD
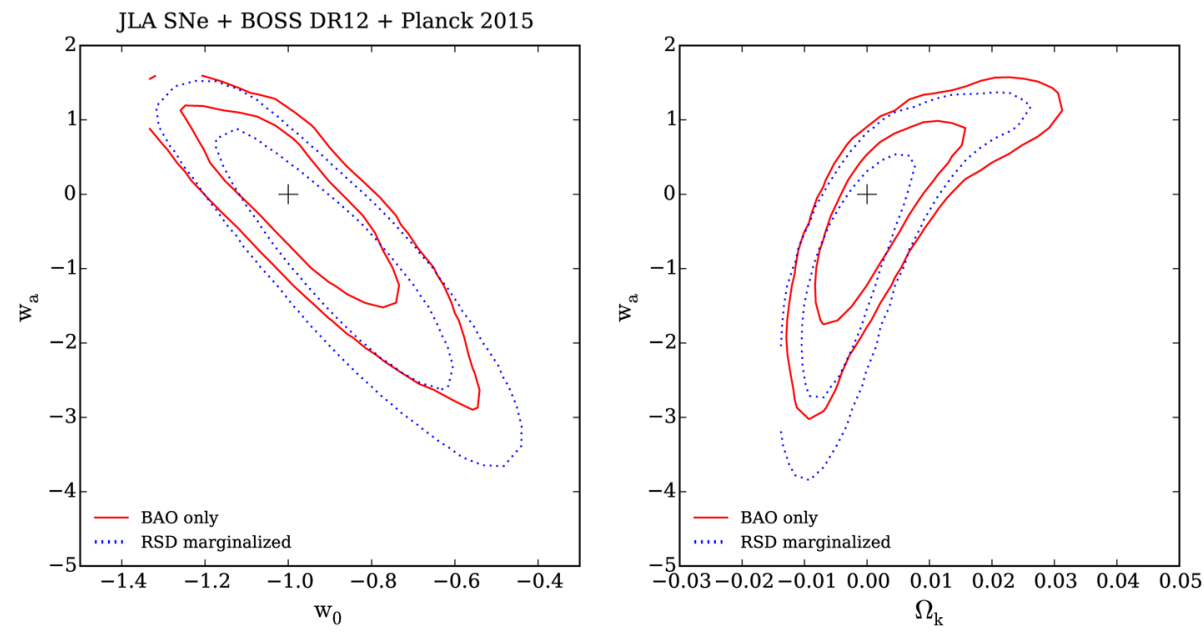

FIG. 2. The joint $68 \%$ and $95 \%$ confidence contours for $\left(w_{a}, w_{0}\right)$ and $\left(w_{a}, \Omega_{k}\right)$ corresponding to Fig. 1, with the same line types.

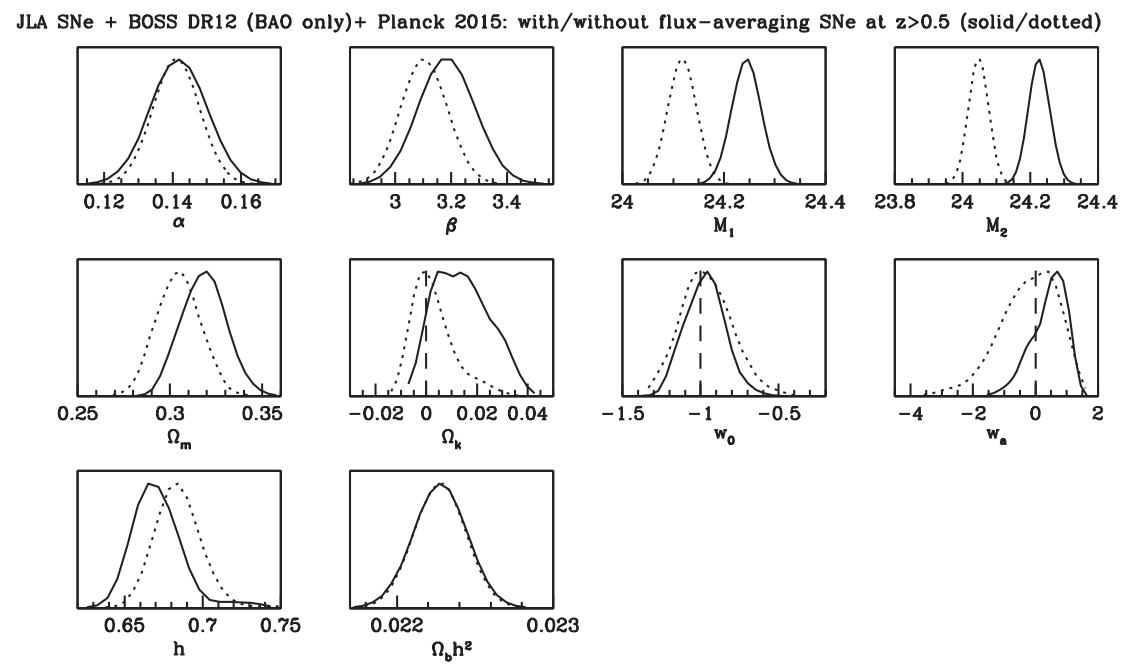

FIG. 3. Marginalized probability distributions of parameters from JLA SNe, galaxy clustering data at $z=0.32$ and $z=0.57$ (BAO only), and Planck 2015 distance priors. The solid and dotted curves correspond to using SNe Ia with and without flux averaging. 

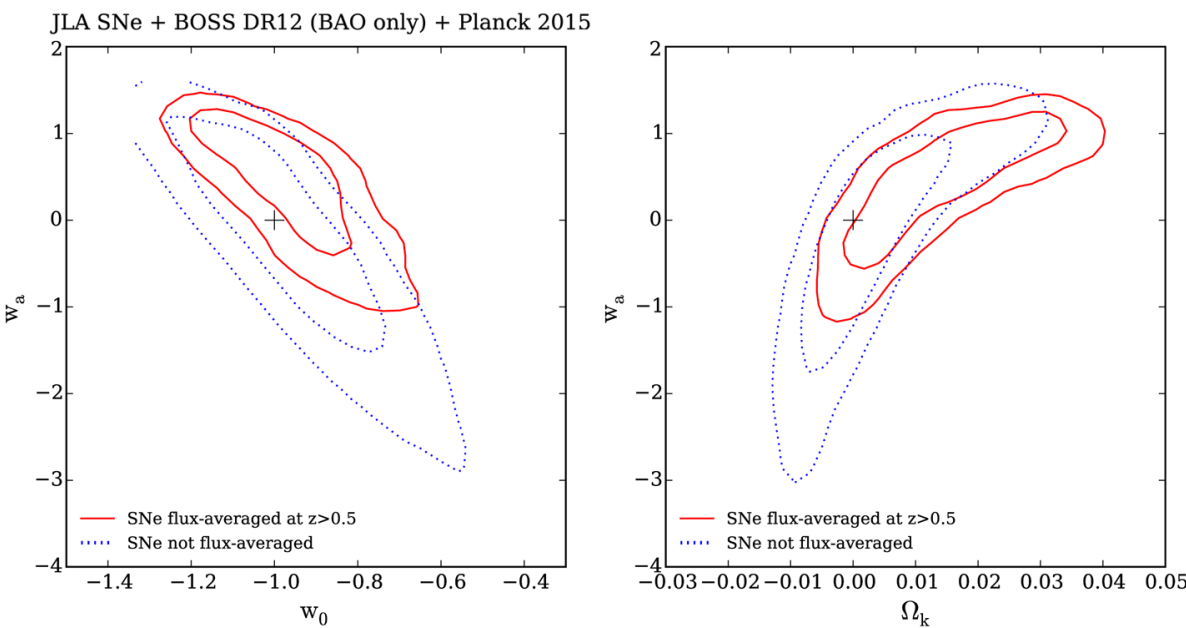

FIG. 4. The joint $68 \%$ and $95 \%$ confidence contours for $\left(w_{a}, w_{0}\right)$ and $\left(w_{a}, \Omega_{k}\right)$ corresponding to Fig. 3 . The solid and dotted curves correspond to using $\mathrm{SNe}$ Ia with and without flux averaging.

marginalized measurements. Figure 6 shows the joint $68 \%$ and $95 \%$ confidence contours for $\left(w_{0.5}, w_{0}\right)$ and $\left(w_{0.5}, \Omega_{k}\right)$ corresponding to Fig. 5, with the same line types. Again, the assumptions made in the analysis of galaxy clustering data have little impact on the combined dark energy constraints.

Figure 7 shows the impact of flux-averaging SNe Ia on the marginalized probability distributions of parameters from the combination of the same data sets as in Fig. 5. The solid and dotted curves correspond to using SNe Ia with and without flux averaging. Figure 8 shows the joint $68 \%$ and $95 \%$ confidence contours for $\left(w_{0.5}, w_{0}\right)$ and $\left(w_{0.5}, \Omega_{k}\right)$ corresponding to Fig. 7 , with the same line types. Flux averaging of $\mathrm{SNe}$ makes an even more dramatic difference in the joint constraints on $\left(w_{0}, w_{0.5}\right)$, compared to those of $\left(w_{0}, w_{a}\right)$.

\section{Constraints on the dark energy density function}

We now examine the dark energy constraints when we allow the dark energy density function, $X(z) \equiv$ $\rho_{X}(z) / \rho_{X}(z=0)$, to be a free function, given by the cubic spline of its value at $z=0,1 / 3,2 / 3,1$, and assuming that $X(z>1)=X(z=1)$.

Figure 9 shows the impact of the galaxy clustering analysis technique on the marginalized probability distributions of parameters from JLA SNe, galaxy clustering data at $z=0.32$ and $z=0.57$ [31,32], and Planck 2015 distance priors presented in this paper (see Sec. II A). The

JLA SNe + BOSS DR12 + Planck 2015: BAO only (solid); RSD marginalized (dotted)
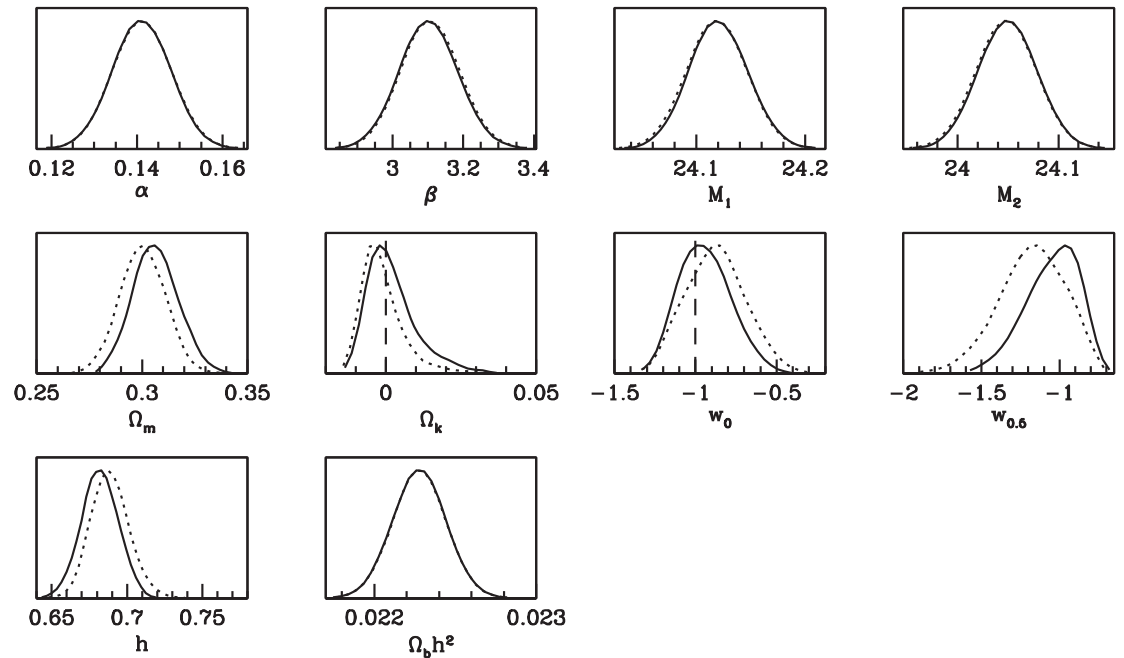

FIG. 5. Marginalized probability distributions of parameters from JLA SNe, galaxy clustering data at $z=0.32$ and $z=0.57$, and Planck 2015 distance priors. The solid and dotted curves correspond to using $H(z)$ and $D_{A}(z)$ measurements from BAO-only measurements, and using those from RSD marginalized measurements. 

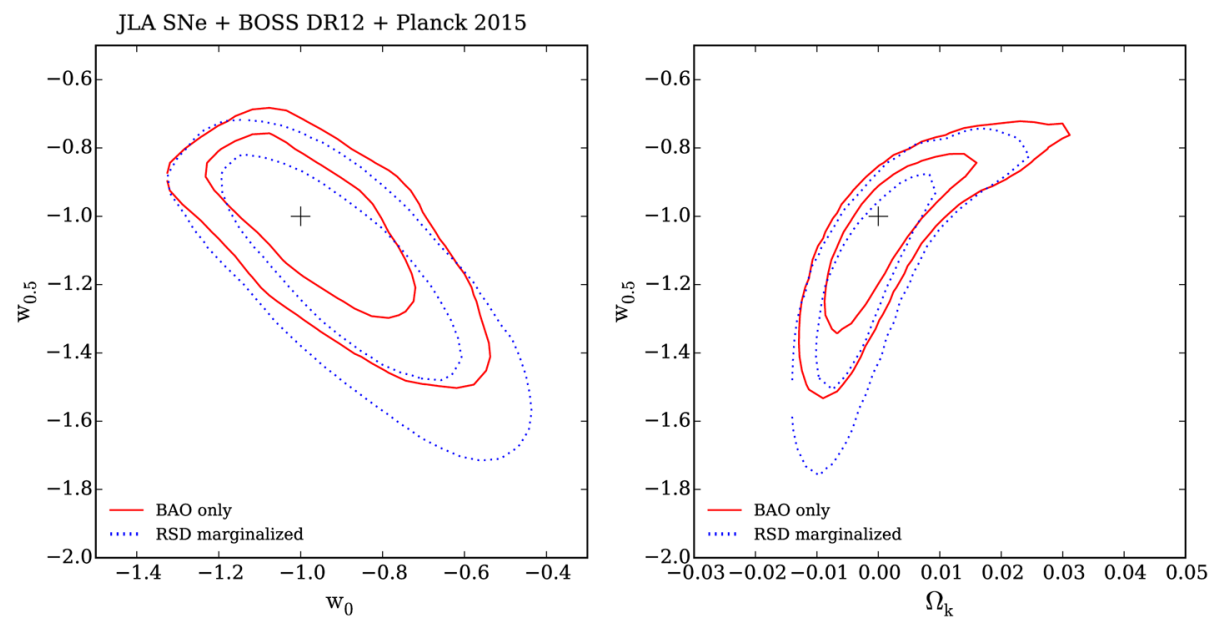

FIG. 6. The joint $68 \%$ and $95 \%$ confidence contours for $\left(w_{0.5}, w_{0}\right)$ and $\left(w_{0.5}, \Omega_{k}\right)$ corresponding to Fig. 5. The solid and dotted curves correspond to using $H(z)$ and $D_{A}(z)$ measurements from BAO-only measurements, and using those from RSD marginalized measurements.

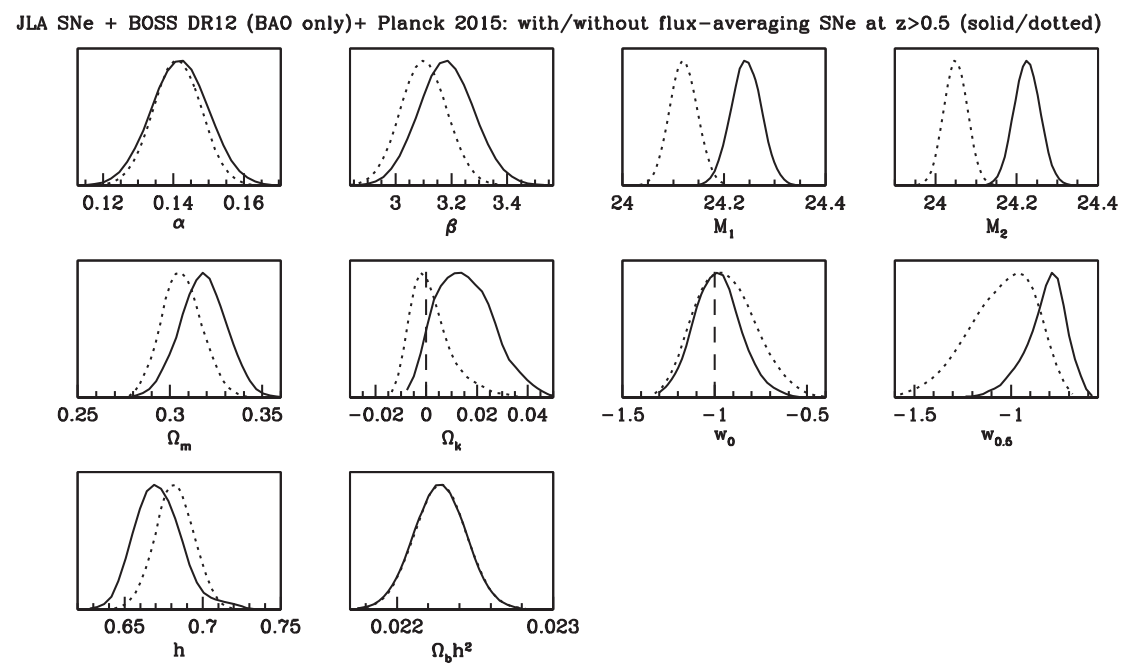

FIG. 7. Marginalized probability distributions of parameters from JLA SNe, galaxy clustering data at $z=0.32$ and $z=0.57$ (BAO only), and Planck 2015 distance priors. The solid and dotted curves correspond to using SNe Ia with and without flux averaging.
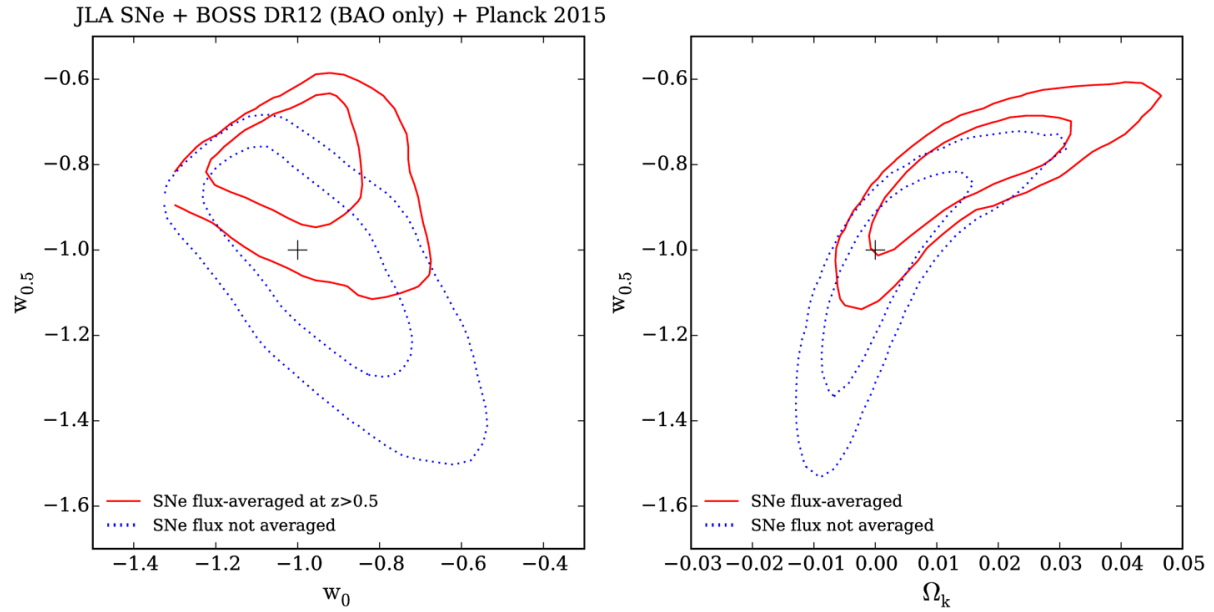

FIG. 8. The joint $68 \%$ and $95 \%$ confidence contours for $\left(w_{0.5}, w_{0}\right)$ and $\left(w_{0.5}, \Omega_{k}\right)$ corresponding to Fig. 7 . The solid and dotted curves correspond to using $\mathrm{SNe}$ Ia with and without flux averaging. 

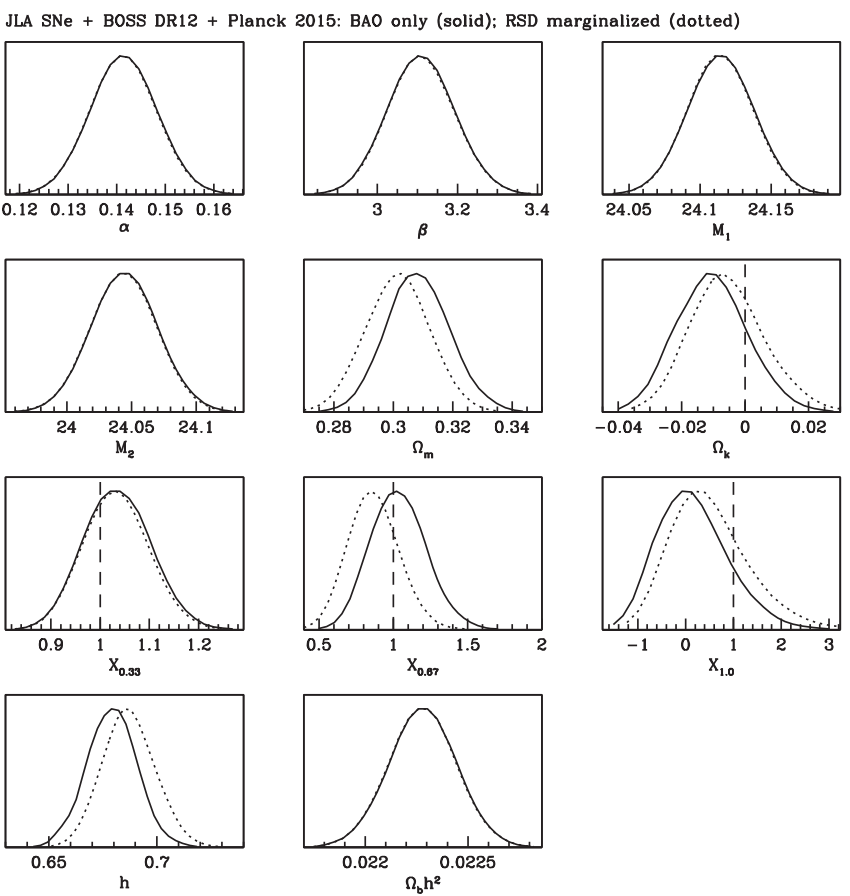

FIG. 9. Marginalized probability distributions of parameters from JLA SNe, galaxy clustering data at $z=0.32$ and $z=0.57$, and Planck 2015 distance priors. The solid and dotted curves correspond to using $H(z)$ and $D_{A}(z)$ measurements from BAOonly measurements, and using those from RSD marginalized measurements.
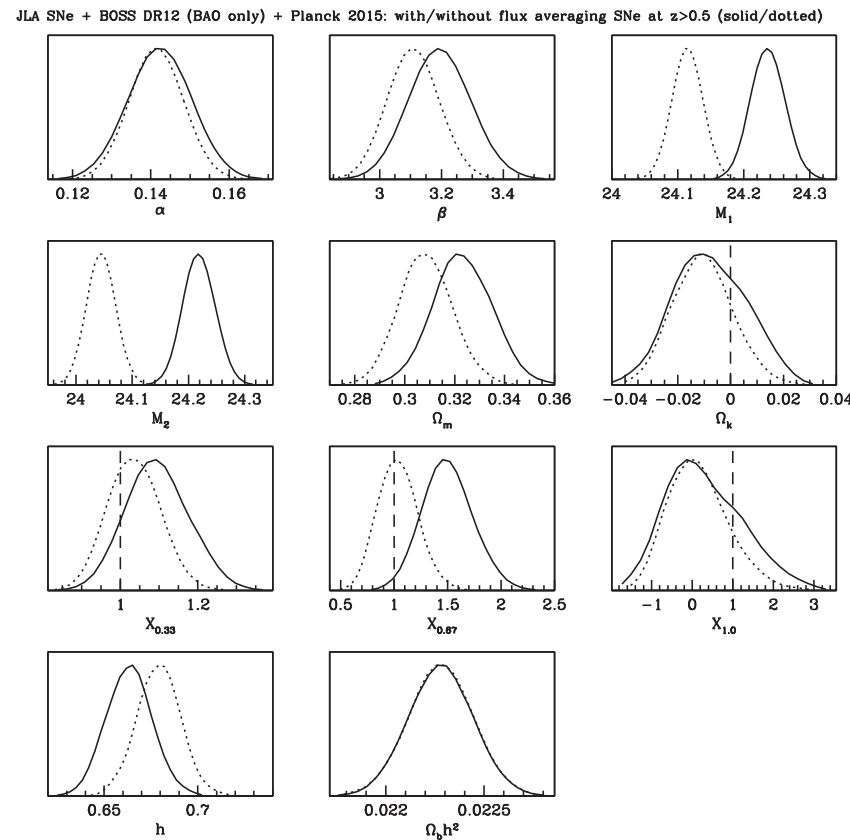

FIG. 10. Marginalized probability distributions of parameters from JLA SNe, galaxy clustering data at $z=0.32$ and $z=0.57$ (BAO only), and Planck 2015 distance priors. The solid and dotted curves correspond to using $\mathrm{SNe}$ Ia with and without flux averaging.

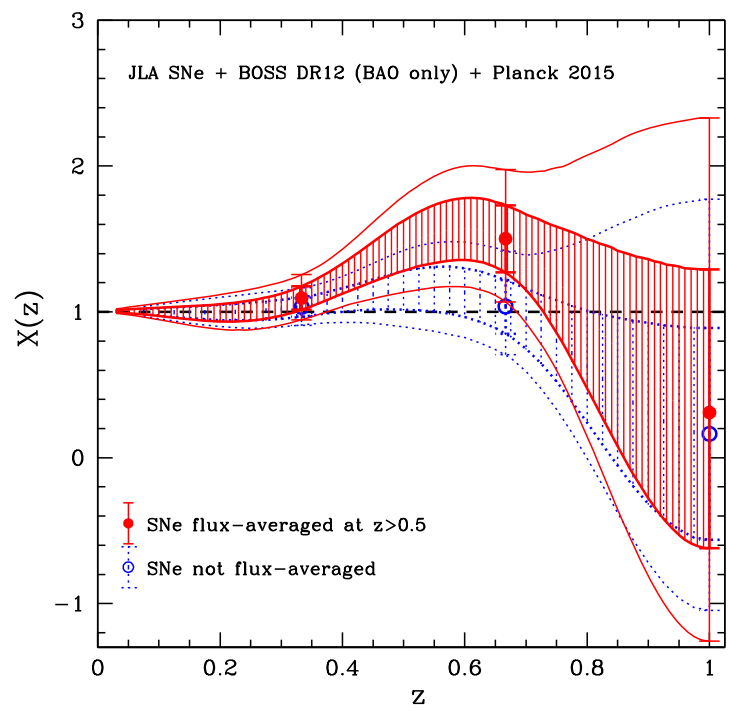

FIG. 11. The dark energy density function $X(z)=\rho_{X}(z) / \rho_{X}(0)$ measured from JLA SNe, galaxy clustering data at $z=0.32$ and $z=0.57$ [31,32], and Planck 2015 distance priors presented in this paper (see Sec. II A). The shaded regions indicate the $68 \%$ confidence region, while the outer envelope indicates the $95 \%$ confidence level. The densely shaded and sparsely shaded regions correspond to using $\mathrm{SNe}$ Ia with and without flux averaging, respectively.

solid and dotted curves correspond to using $H(z)$ and $D_{A}(z)$ measurements from BAO-only measurements, and using those from RSD marginalized measurements. Figure 10 shows the impact of flux-averaging the $\mathrm{SNe}$ Ia on the marginalized probability distributions of parameters from the same combination of data sets. The solid and dotted curves correspond to using SNe Ia with and without flux averaging. Again, we find that the assumptions made in the galaxy clustering data analysis have little impact on the combined dark energy constraints, while flux averaging of SNe Ia has a significant impact on these constraints.

Figure 11 shows the dark energy density function $X(z)=\rho_{X}(z) / \rho_{X}(0)$ measured from JLA SNe, galaxy clustering data at $z=0.32$ and $z=0.57$ [31,32], and Planck 2015 distance priors presented in this paper (see Sec. II A). The shaded regions indicate the $68 \%$ confidence region, while the outer envelope indicates the $95 \%$ confidence level. The densely shaded and sparsely shaded regions correspond to using $\mathrm{SNe}$ Ia with and without flux averaging, respectively. Flux averaging has the most significant effect here-the measured $X(z)$ deviates from $X(z)=1(w=-1)$ at more than a $95 \%$ confidence level for $0.4 \lesssim z \lesssim 0.7$.

\section{DISCUSSION AND SUMMARY}

We have explored the existence of unknown systematic uncertainties in the current SN Ia and galaxy clustering data, with the help of the latest CMB distance priors. We 
use the JLA set of 740 SNe Ia from Betoule et al. (2014) [22], and the measurements of $H(z)$ and $D_{A}(z)$ at $z=0.32$ and $z=0.57$ from BOSS DR12 data by Gil-Marin et al. (2016) [31,32]. We have derived the CMB distance priors from Planck 2015 data, in the form of the mean values and covariance matrix of $\left\{l_{a}, R, \Omega_{b} h^{2}, n_{s}\right\}$, which give an efficient summary of Planck data in the context of dark energy constraints [see Eqs. (13)-(16)].

It is remarkable that the Planck distance priors that we have derived from the 2015 Planck data have uncertainties that are within $10 \%$ of the forecasted errors for Planck by Mukherjee et al. (2008) [34]. This indicates that Planck has achieved its forecasted precision in cosmological constraints. We note that Huang, Wang, and Wang [35] independently derived similar but slightly different constraints from Planck 2015 data at approximately the same time.

We find that the combined dark energy constraints are insensitive to the assumptions made in the galaxy clustering measurements (whether they are for BAO only [31] or marginalized over RSD [32]), independent of the dark energy parametrization used (see Figs. 1, 2, 5, 6, 9). We note that the published BAO-only constraints in Ref. [31] differ from those in the earlier arXiv version, and are closer to the RSD marginalized constraints in Ref. [32]. This is reassuring, as it indicates that as the analysis of galaxy clustering data becomes more accurate and robust, the systematic uncertainties are reduced.

On the other hand, we find that flux-averaging $\mathrm{SNe}$ Ia at $z \geq 0.5$ significantly tightens the dark energy constraints, and excludes $w=-1$ at greater than $68 \%$ confidence level (see Figs. 3, 4, 7, 8, 10, 11). Flux averaging has the most significant effect when we allow the dark energy density function $X(z)=\rho_{X}(z) / \rho_{X}(0)$ to be a free function given by the cubic spline of its values at $z=0, \frac{1}{3}, \frac{2}{3}, 1$; the measured $X(z)$ deviates from $X(z)=1(w=-1)$ at more than a 95\% confidence level for $0.4 \lesssim z \lesssim 0.7$ (see Fig. 11). This is somewhat surprising, since for SN data with redshiftdependent systematic biases that are negligible compared to statistical errors, flux averaging of $\mathrm{SNe}$ should give somewhat less stringent constraints on dark energy [25]. Since flux averaging reduces the bias in the $\mathrm{SN}$ distance measurements [28], this may be an indication that we have arrived in the era when the $\mathrm{SN}$ distance measurements are limited by systematic uncertainties.

Identifying and correctly modeling systematic effects will be key in illuminating the nature of dark energy. Future dark energy surveys from space [36-39] will be designed to minimize systematic uncertainties. We can expect dramatic progress in the next decade in our quest to shed light on dark energy.

\section{ACKNOWLEDGMENTS}

We are grateful to Rick Kessler and Ranga Chary for helpful discussions, and to Alex Merson for providing python scripts for making 2D contour plots. We acknowledge the use of the Planck data arXiv and CosmoMC.
[1] A. G. Riess et al., Astron. J. 116, 1009 (1998).

[2] S. Perlmutter et al., Astrophys. J. 517, 565 (1999).

[3] E. J. Copeland, M. Sami, and S. Tsujikawa, Int. J. Mod. Phys. D 15, 1753 (2006).

[4] P. Ruiz-Lapuente, Classical Quantum Gravity 24, R91 (2007).

[5] B. Ratra and M. S. Vogeley, Publ. Astron. Soc. Pac. 120, 235 (2008).

[6] J. Frieman, M. Turner, and D. Huterer, Annu. Rev. Astron. Astrophys. 46, 385 (2008).

[7] R. R. Caldwell and M. Kamionkowski, Annu. Rev. Nucl. Part. Sci. 59, 397 (2009).

[8] J.-P. Uzan, Gen. Relativ. Gravit. 42, 2219 (2010).

[9] Y. Wang, Dark Energy (Wiley-VCH, Weinheim, Germany, 2010).

[10] M. Li, X.-D. Li, S. Wang, and Y. Wang, Commun. Theor. Phys. 56, 525 (2011).

[11] D. H. Weinberg, M. J. Mortonson, D. J. Eisenstein, C. Hirata, A. G. Riess, and E. Rozo, Phys. Rep. 530, 87 (2013).

[12] Y. Wang, J. Cosmol. Astropart. Phys. 05 (2008) 021.

[13] F. Simpson and J. A. Peacock, Phys. Rev. D 81, 043512 (2010).
[14] M. Chevallier and D. Polarski, Int. J. Mod. Phys. D 10, 213 (2001).

[15] Y. Wang, Phys. Rev. D 77, 123525 (2008).

[16] Y. Wang and P. Mukherjee, Phys. Rev. D 76, 103533 (2007).

[17] H. Li, J.-Q. Xia, G.-B. Zhao, Z.-H. Fan, and X. Zhang, Astrophys. J. 683, L1 (2008).

[18] L. Page et al., Astrophys. J. Suppl. Ser. 148, 233 (2003).

[19] W. Hu and N. Sugiyama, Astrophys. J. 471, 542 (1996).

[20] D. Eisenstein and W. Hu, Astrophys. J. 496, 605 (1998).

[21] Y. Wang, C.-H. Chuang, and P. Mukherjee, Phys. Rev. D 85, 023517 (2012).

[22] M. Betoule et al., Astron. Astrophys. 568, A22 (2014).

[23] A. Conley et al., Astrophys. J. Suppl. Ser. 192, 1 (2011).

[24] L. Hui and P. B. Green, Phys. Rev. D 73, 123526 (2006).

[25] Y. Wang, Astrophys. J. 536, 531 (2000).

[26] Y. Wang and P. Mukherjee, Astrophys. J. 606, 654 (2004).

[27] Y. Wang, J. Cosmol. Astropart. Phys. 03 (2005) 005.

[28] Y. Wang and M. Tegmark, Phys. Rev. D 71, 103513 (2005).

[29] D. Sarkar, A. Amblard, D. E. Holz, and A. Cooray, Astrophys. J. 678, 1 (2008).

[30] C.-H. Chuang and Y. Wang, Mon. Not. R. Astron. Soc. 426, 226 (2012). 
[31] H. Gil-Marin et al., Mon. Not. R. Astron. Soc. 460, 4210 (2016).

[32] H. Gil-Marin et al., Mon. Not. R. Astron. Soc. 460, 4188 (2016).

[33] A. Lewis and S. Bridle, Phys. Rev. D 66, 103511 (2002).

[34] P. Mukherjee, M. Kunz, D. Parkinson, and Y. Wang, Phys. Rev. D 78, 083529 (2008).
[35] Q. G. Huang, K. Wang, and S. Wang, J. Cosmol. Astropart. Phys. 12 (2015) 022.

[36] A. Crotts et al., arXiv:astro-ph/0507043.

[37] A. Cimatti et al., Exp. Astron. 23, 39 (2009).

[38] R. Laureijs et al., arXiv:1110.3193.

[39] D. Spergel et al., arXiv:1503.03757. 